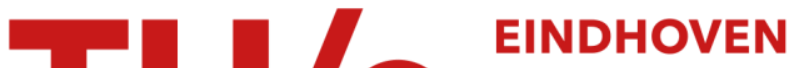 UNIVERSITY OF TECHNOLOGY
}

\section{A random environment for linearly edge-reinforced random walks on infinite graphs}

\section{Citation for published version (APA):}

Rolles, S. W. W., \& Merkl, F. (2005). A random environment for linearly edge-reinforced random walks on infinite graphs. (Report Eurandom; Vol. 2005051). Eurandom.

\section{Document status and date:}

Published: 01/01/2005

\section{Document Version:}

Publisher's PDF, also known as Version of Record (includes final page, issue and volume numbers)

\section{Please check the document version of this publication:}

- A submitted manuscript is the version of the article upon submission and before peer-review. There can be important differences between the submitted version and the official published version of record. People interested in the research are advised to contact the author for the final version of the publication, or visit the $\mathrm{DOI}$ to the publisher's website.

- The final author version and the galley proof are versions of the publication after peer review.

- The final published version features the final layout of the paper including the volume, issue and page numbers.

Link to publication

\section{General rights}

Copyright and moral rights for the publications made accessible in the public portal are retained by the authors and/or other copyright owners and it is a condition of accessing publications that users recognise and abide by the legal requirements associated with these rights.

- Users may download and print one copy of any publication from the public portal for the purpose of private study or research.

- You may not further distribute the material or use it for any profit-making activity or commercial gain

- You may freely distribute the URL identifying the publication in the public portal.

If the publication is distributed under the terms of Article $25 \mathrm{fa}$ of the Dutch Copyright Act, indicated by the "Taverne" license above, please follow below link for the End User Agreement:

www.tue.nl/taverne

\section{Take down policy}

If you believe that this document breaches copyright please contact us at:

openaccess@tue.nl

providing details and we will investigate your claim. 


\title{
A Random Environment \\ for Linearly Edge-Reinforced Random Walks on Infinite Graphs
}

\author{
Franz Merkl ${ }^{1}$
}

Silke W.W. Rolles ${ }^{2}$

August 19, 2005

\begin{abstract}
We consider linearly edge-reinforced random walk on an arbitrary locally finite connected graph. It is shown that the process has the same distribution as a random walk in a time-independent random environment given by strictly positive weights on the edges. Furthermore, we prove bounds for the random environment, uniform, among others, in the size of the graph. ${ }^{34}$
\end{abstract}

\section{Introduction}

Linearly edge-reinforced random walk (errw) is the following model: Consider a locally finite connected graph $G=(V, E)$ with vertex set $V$ and edge set $E$. The edges are undirected. A random walker moves randomly on the vertices of the graph, traversing an edge between each discrete time $t=0,1,2, \ldots$. Let $X_{t}$ denote the random location of the random walker at time $t$. At time 0 , the random walker starts in a distinguished vertex $X_{0}=\mathbf{0} \in V$. We realize the $X_{t}$ as canonical projections defined on the set $\Omega_{\mathbf{0}} \subseteq V^{\mathbb{N}_{0}}$ of all admissible paths.

The random walk $\left(X_{t}\right)_{t \in \mathbb{N}_{0}}$ is non-Markovian. The relevant memory of the random walker is encoded in random weights $w_{e}(t), e \in E, t \in \mathbb{N}_{0}$, which change with time. Initially, the weights take prescribed values $w_{e}(0):=a_{e}>0, e \in E$, possibly depending on the edge $e$. Each time the random walker traverses the edge $e=\{u, v\} \in E$, the weight of $e$ is increased by 1 , and the weight of all the other edges $e^{\prime} \in E \backslash\{e\}$ remain unchanged. In other words:

$$
w_{e}(t):=a_{e}+\sum_{s=1}^{t} 1_{e}\left(\left\{X_{s-1}, X_{s}\right\}\right), \quad\left(e \in E, t \in \mathbb{N}_{0}\right) .
$$

The random weights $w_{e}(t)$, representing the memory of the random walker at time $t$, determine the transition probabilities of the random walker as follows: For any $u \in V$,

\footnotetext{
${ }^{1}$ Mathematical Institute, University of Munich, Theresienstr. 39, D-80333 Munich, Germany. e-mail: merkl@mathematik.uni-muenchen.de

${ }^{2}$ Eindhoven University of Technology, Department of Mathematics, P.O. Box 513, 5600 MB Eindhoven, The Netherlands. e-mail: srolles@win.tue.nl

${ }^{3} \mathrm{MSC} 2000$ subject classifications: Primary 60K35; secondary 60K37.

${ }^{4}$ Key words: Reinforced random walk, random environment.
} 
one has

$$
P_{\mathbf{0}, a}^{G}\left[X_{t+1}=u \mid X_{0}, \ldots, X_{t}\right]=\frac{w_{\left\{X_{t}, u\right\}}(t)}{w_{X_{t}}(t)} \quad \text { whenever }\left\{X_{t}, u\right\} \in E,
$$

where for $v \in V$, we set

$$
w_{v}(t):=\sum_{e \ni v} w_{e}(t) \quad \text { and } \quad a=\left(a_{e}\right)_{e \in E}
$$

Whenever $\left\{X_{t}, u\right\} \notin E$, then

$$
P_{\mathbf{0}, a}^{G}\left[X_{t+1}=u \mid X_{0}, \ldots, X_{t}\right]=0 .
$$

In other words, the transition probabilities are proportional to the weight of the traversed edge at that time. For an overview of the history of the model see e.g. [MR05c].

Linearly edge-reinforced random walk is partially exchangeable in the following sense: Two finite paths with the same starting point are traversed with the same probability by the reinforced random walker provided every (undirected) edge is traversed the same number of times in both paths. If the reinforced random walk is recurrent, that is, if it returns to its starting point infinitely often with probability one, then a de Finetti theorem for Markov chains due to Diaconis and Freedman [DF80] implies that the reinforced random walk is a mixture of Markov chains. In particular, this applies to finite graphs. Even more, on any finite graph, the reinforced random walk is a mixture of reversible Markov chains; this follows e.g. by a de Finetti theorem for reversible Markov chains (see [Rol03]). However, on many infinite, locally finite graphs with some initial weights, edge-reinforced random walk is not recurrent, and on some infinite, locally finite graphs, including $\mathbb{Z}^{d}$ for $d \geq 2$, it is not known whether edge-reinforced random walk is recurrent. In this paper, we prove that for any locally finite graph and any initial weights, edge-reinforced random walk is a mixture of Markov chains, irrespectively whether it is recurrent or not. The Markov chains are defined in terms of random, time-independent weights on the (undirected) edges. For finite graphs, there is an explicit but complicated formula for the joint distribution of these random weights. They show a complicated dependence structure. This description was first given by Coppersmith and Diaconis in [CD86] and refined by Keane and Rolles in [KR00]. It has already been useful to analyze edge-reinforced random walk on certain infinite graphs, including ladders of arbitrary width, by taking the infinite volume limit of finite subgraphs; see [MR05b], [Rol05], and [MR05a]. However, in this paper, we do not make use of the explicit form of the mixing measure for finite graphs.

\section{Results}

For ordinary (Markovian) random walks, the following is known: If the random walker returns to its starting point a.s. at least once, then it also returns a.s. infinitely often. For general non-Markovian random walks, this breaks down. However, for linearly edgereinforced random walks, the implication is still true, as the following theorem shows: 
Theorem 2.1 For the edge-reinforced random walk on any locally finite connected graph, the following statements are equivalent:

(a) The edge-reinforced random walker returns to its starting point with probability one.

(b) The edge-reinforced random walker returns to its starting point infinitely often with probability one.

(c) With probability one, every vertex is visited at least twice by the edge-reinforced random walker.

(d) The edge-reinforced random walker visits all vertices infinitely often with probability one.

For $x=\left(x_{e}\right)_{e \in E} \in \mathbb{R}_{+}^{E}$, let $Q_{\mathbf{0}, x}^{G}$ denote the distribution of the Markov chain on $G$ over $\Omega_{\mathbf{0}}$ with starting vertex $\mathbf{0}$ and time-independent transition probabilities

$$
Q_{\mathbf{0}, x}^{G}\left[X_{t+1}=u^{\prime} \mid X_{t}=u\right]=\frac{x_{\left\{u, u^{\prime}\right\}}}{\sum_{e \in E: u \in e} x_{e}} 1_{\left\{\left\{u, u^{\prime}\right\} \in E\right\}}
$$

The following theorem generalizes the known representation of linearly edge-reinforced random walk on finite graphs to general locally finite, possibly infinite graphs.

Theorem 2.2 For edge-reinforced random walk on any locally finite graph $G$ with any starting vertex $\mathbf{0}$ and any initial edge weights $a=\left(a_{e}\right)_{e \in E} \in \mathbb{R}_{+}^{E}$, there exists a probability measure $\mathbb{Q}_{0, a}^{G}$ on the set $(0, \infty)^{E}$ of strictly positive edge weights such that for all events $A \subseteq \Omega_{0}$, one has

$$
P_{\mathbf{0}, a}^{G}[A]=\int_{\mathbb{R}_{+}^{E}} Q_{\mathbf{0}, x}^{G}[A] \mathbb{Q}_{\mathbf{0}, a}^{G}(d x),
$$

i.e. the edge-reinforced random walk has the same distribution as a random walk in a random environment given by (time-independent) random weights on the edges.

We prove bounds for the distribution of the transition probabilities of edge-reinforced random walk. These estimates hold for any locally finite, possibly infinite graph and any initial weights. They depend only on the local structure of the graph and on the initial weights nearby.

Theorem 2.3 For edge-reinforced random walk on any locally finite graph $G$ with any starting vertex $\mathbf{0}$ and any initial edge weights a, there is a measure $\mathbb{Q}_{\mathbf{0}, a}^{G}$ with the properties specified in Theorem 2.2, such that the following holds: For any vertex $v$ and any edge $e$ incident to $v$, there are constants $c_{1}=c_{1}\left(a_{v}, a_{e}\right)>0$ and $c_{2}=c_{2}\left(a_{v}, a_{e}\right)>0$, depending continuously only on $a_{v}$ and $a_{e}, a_{v} \geq a_{e}>0$, but not depending on any other details of $G, \mathbf{0}$, and $a$, such that for all $\varepsilon>0$, the estimates

$$
\mathbb{Q}_{\mathbf{0}, a}^{G}\left[\frac{x_{e}}{x_{v}} \leq \varepsilon\right] \leq c_{1} \varepsilon^{a_{e} / 2} \quad \text { and } \quad \mathbb{Q}_{0, a}^{G}\left[\frac{x_{e}}{x_{v}} \geq 1-\varepsilon\right] \leq c_{2} \varepsilon^{\left(a_{v}-a_{e}\right) / 2}
$$

hold. 
The random variables $x_{e} / x_{f} ; e, f \in E$ are tight with algebraically bounded tails, uniformly in the choice of the graph $G$, provided that the graph distance from $e$ to $f$ is bounded, and provided that the initial weights are bounded and bounded away from 0 .

More precisely:

Theorem 2.4 For all compact sets $K \subset(0, \infty)$, there exists a constant $c_{3}(K)>0$, such that for all locally finite graphs $G=(V, E)$, any starting vertex $\mathbf{0}$ and all initial edge weights $a=\left(a_{e}\right)_{e \in E}$, there is a measure $\mathbb{Q}_{\mathbf{0}, a}^{G}$ with the properties specified in Theorems 2.2 and 2.3, such that the following holds: For all edges $e, f \in E$, for all paths

$$
\stackrel{e=e_{0}}{\longrightarrow} v_{1} \stackrel{e_{1}}{\longrightarrow} v_{2} \stackrel{e_{2}}{\longrightarrow} \ldots \stackrel{e_{l-1}}{\longrightarrow} v_{l} \stackrel{e_{l}=f}{\longrightarrow}
$$

of length $l+1$ from e to $f$ with the property $a_{e_{i}} \in K$ and $a_{v_{j}}=\sum_{e^{\prime} \ni v_{j}} a_{e^{\prime}} \in K(i=0, \ldots, l$; $j=1, \ldots, l)$ and for all $M>0$, one has

$$
\mathbb{Q}_{\mathbf{0}, a}^{G}\left[x_{e} \geq M x_{f}\right] \leq c_{3}(K) l M^{-\gamma}
$$

where

$$
\gamma=\frac{1}{2 l} \min \left\{a_{e_{i}}: 1 \leq i \leq l\right\}
$$

In general, it is not known whether the measure $\mathbb{Q}_{\mathbf{0}, a}^{G}$ is unique. If the underlying graph is of the form $\mathbb{Z} \times T$ with a finite tree $T$ and the initial weights are large, then, up to multiplication of all $x_{e}$ by the same constant, there is a unique measure $\mathbb{Q}_{\mathbf{0}, a}^{G}$ satisfying (2.2) for all events $A$; see Theorem 2.4 in [MR05a]. Furthermore, in that case, $\sum_{e \in E} x_{e}<\infty$ holds $\mathbb{Q}_{0, a}^{G}$-almost surely.

The bounds in Theorems 2.3 and 2.4 are first proven on finite graphs. In the sense of convex order, we bound the jump probability distribution for edge-reinforced random walk by transition probabilities of suitable Polya urn models. The notion of convex order plays an important role in our argument. Therefore, we review the basic properties of convex order in Section 3. The comparison with Polya urn models is a local construction. It therefore yields uniform bounds in the size of the graph. We approximate an infinite locally finite graph by growing finite pieces, using compactness and tightness arguments. The uniformity of the estimates is essential in this approximation to get tightness.

\section{Preliminaries}

In this section, we collect just the basic properties of convex order that we need.

Definition 3.1 Let $X$ and $Y$ be random variables with finite expectation, not necessarily defined on the same probability space. We say that $X$ and $Y$ are in convex order, in symbols $X \triangleleft Y$, if there are random variables $X_{1} \stackrel{d}{=} X$ and $Y_{1} \stackrel{d}{=} Y$ defined on a common probability space $(\Omega, \mathcal{A}, P)$ such that $\left(X_{1}, Y_{1}\right)$ is a 1-step martingale, i.e., if there is a $\sigma$-field $\mathcal{F} \subseteq \mathcal{A}$ such that $X_{1}$ is $\mathcal{F}$-measurable and

$$
X_{1}=E_{P}\left[Y_{1} \mid \mathcal{F}\right]
$$


holds a.s. The relation $X \triangleleft Y$ depends only on the laws $\mathcal{L}_{X}$ and $\mathcal{L}_{Y}$ of $X$ and $Y$; we therefore write also $\mathcal{L}_{X} \triangleleft \mathcal{L}_{Y}$.

Note that we may always replace $\mathcal{F}$ by $\sigma\left(X_{1}\right)$ in $(3.1)$.

Lemma 3.2 (a) The relation $\triangleleft$ is transitive.

(b) Assume that $\left(X_{n}\right)_{n \in \mathbb{N}}$ and $\left(Y_{n}\right)_{n \in \mathbb{N}}$ are uniformly integrable martingales with respect to filtrations $\left(\mathcal{F}_{n}\right)_{n \in \mathbb{N}}$ and $\left(\mathcal{G}_{n}\right)_{n \in \mathbb{N}}$, respectively, not necessarily defined on the same probability space. Let $X$ and $Y$ denote the a.s. limit of $\left(X_{n}\right)_{n}$ and $\left(Y_{n}\right)_{n}$, respectively. Assume that for all $n \in \mathbb{N}$, the relation $X_{n} \triangleleft Y_{n}$ holds. Then $X \triangleleft Y$ also holds.

(c) Let $\mu_{1}, \ldots, \mu_{n}$ and $\nu_{1}, \ldots, \nu_{n}$ be distributions on $\mathbb{R}$ such that $\mu_{i} \triangleleft \nu_{i}$ holds for all $i=1, \ldots, n$. Let $p_{i}, i=1, \ldots, n$, be nonnegative numbers with sum 1 . Then

$$
\sum_{i=1}^{n} p_{i} \mu_{i} \triangleleft \sum_{i=1}^{n} p_{i} \nu_{i}
$$

also holds.

(d) Let $X \triangleleft Y$. For any convex function $f: I \rightarrow \mathbb{R}$, defined at least on an interval $I \subseteq \mathbb{R}$ containing the range of $X$ and $Y$, such that $E[f(Y)]<\infty$ is valid, the inequality

$$
E[f(X)] \leq E[f(Y)]
$$

holds.

Remark. As a consequence of Strassen's theorem (see e.g. Theorem 2 in Strassen's classical paper [Str65]), the converse of part (d) is also true. This theorem could be used to prove the lemma. However, in this paper, we rather base the proof of the lemma on more direct, elementary arguments, using only Jensen's inequality, i.e. the trivial direction of Strassen's theorem.

\section{Proof.}

(a) Assume that $X \triangleleft Y$ and $Y \triangleleft Z$. Let $X_{1} \stackrel{d}{=} X$ and $Y_{1} \stackrel{d}{=} Y$ be random variables on a common probability space $\left(\Omega_{1}, \mathcal{A}_{1}, P_{1}\right)$, such that $X_{1}=E_{P_{1}}\left[Y_{1} \mid X_{1}\right]$ holds. Let $P_{1}\left[Y_{1} \in \cdot \mid X_{1}=\cdot\right]$ denote a regular conditional distribution of $Y_{1}$ conditional on $X_{1}$, i.e. it is a stochastic kernel defined on $\mathbb{R} \times \mathcal{B}(\mathbb{R})$ such that for all $A, B \in \mathcal{B}(\mathbb{R})$, one has

$$
P_{1}\left[X_{1} \in A, Y_{1} \in B\right]=\int_{A} P_{1}\left[Y_{1} \in B \mid X_{1}=x\right] \mathcal{L}_{X}(d x)
$$

Similarly, let $Y_{2} \stackrel{d}{=} Y$ and $Z_{2} \stackrel{d}{=} Z$ be random variables on a common probability space $\left(\Omega_{2}, \mathcal{A}_{2}, P_{2}\right)$, such that $Y_{2}=E_{P_{2}}\left[Z_{2} \mid Y_{2}\right]$ holds. Let $P_{2}\left[Z_{2} \in \cdot \mid Y_{2}=\cdot\right]$ denote a regular conditional distribution of $Z_{2}$ conditional on $Y_{2}$. Let $P_{3}$ be the law of a time-inhomogeneous Markov chain with 3 time points, starting distribution $\mathcal{L}_{X}$, first 
transition kernel $P_{1}\left[Y_{1} \in \cdot \mid X_{1}=\cdot\right]$, and second transition kernel $P_{2}\left[Z_{2} \in \cdot \mid Y_{2}=\cdot\right]$, i.e. $P_{3}$ is the probability measure on $\mathcal{B}\left(\mathbb{R}^{3}\right)$ which fulfills

$$
P_{3}[A \times B \times C]=\int_{A} \int_{B} P_{2}\left[Z_{2} \in C \mid Y_{2}=y\right] P\left[Y_{1} \in d y \mid X_{1}=x\right] \mathcal{L}_{X}(d x)
$$

for all cylinder events $A \times B \times C \subseteq \mathbb{R}^{3}$. Let $X_{3}, Y_{3}$, and $Z_{3}$ denote the projections to the first, second, and third coordinate of $\mathbb{R}^{3}$, respectively. By construction, with respect to $P_{3}, X_{3} \stackrel{d}{=} X, Y_{3} \stackrel{d}{=} Y, Z_{3} \stackrel{d}{=} Z$, and we have $P_{3}$-a.s.:

$$
E_{P_{3}}\left[Z_{3} \mid X_{3}, Y_{3}\right]=E_{P_{3}}\left[Z_{3} \mid Y_{3}\right]=Y_{3}
$$

and therefore

$$
E_{P_{3}}\left[Z_{3} \mid X_{3}\right]=E_{P_{3}}\left[E_{P_{3}}\left[Z_{3} \mid X_{3}, Y_{3}\right] \mid X_{3}\right]=E_{P_{3}}\left[Y_{3} \mid X_{3}\right]=X_{3}
$$

This shows $X \triangleleft Z$.

(b) Fix $n \in \mathbb{N}$. Since the martingale $\left(Y_{m}\right)_{m \in \mathbb{N}}$ is uniformly integrable, it also converges in $L^{1}$ to $Y$, and we have $E\left[Y \mid \mathcal{G}_{n}\right]=Y_{n}$ a.s.; in particular $Y_{n} \triangleleft Y$ holds. Since $X_{n} \triangleleft Y_{n}$ holds by assumption, we conclude $X_{n} \triangleleft Y$. Therefore, we can take $X_{n}^{\prime} \stackrel{d}{=} X_{n}$ and $Y_{n}^{\prime} \stackrel{d}{=} Y$ on some common probability space $\left(\Omega_{n}, \mathcal{A}_{n}, P_{n}\right)$ such that $X_{n}^{\prime}=E_{P_{n}}\left[Y_{n}^{\prime} \mid X_{n}^{\prime}\right]$. Hence, for any bounded continuous function $f: \mathbb{R} \rightarrow \mathbb{R}$, the identity

$$
E_{P_{n}}\left[f\left(X_{n}^{\prime}\right) X_{n}^{\prime}\right]=E_{P_{n}}\left[f\left(X_{n}^{\prime}\right) Y_{n}^{\prime}\right]
$$

holds. Since $\left(X_{n}^{\prime}\right)_{n \in \mathbb{N}}$ is uniformly integrable, $\left(X_{n}^{\prime}, Y_{n}^{\prime}\right)_{n \in \mathbb{N}}$ is tight. Thus, there exists a subsequence $\left(X_{n_{k}}^{\prime}, Y_{n_{k}}^{\prime}\right)_{k \in \mathbb{N}}$ that converges weakly to a limit $\left(X^{\prime}, Y^{\prime}\right)$. Taking the limit as $k \rightarrow \infty$ and using uniform integrability yields

$$
E\left[f\left(X^{\prime}\right) X^{\prime}\right]=E\left[f\left(X^{\prime}\right) Y^{\prime}\right]
$$

which is equivalent to $X^{\prime}=E\left[Y^{\prime} \mid X^{\prime}\right]$. Since by construction $\mathcal{L}_{X^{\prime}}=\mathcal{L}_{X}$ (recall that $X_{n} \rightarrow X$ almost surely and hence in distribution) and $\mathcal{L}_{Y^{\prime}}=\mathcal{L}_{Y}$, we have shown that $X \triangleleft Y$.

(c) For $1 \leq i \leq n$, let $X_{i}$ and $Y_{i}$ be random variables on some probability space $\left(\Omega_{i}, \mathcal{A}_{i}, P_{i}\right)$ with $\mathcal{L}_{X_{i}}=\mu_{i}$ and $\mathcal{L}_{Y_{i}}=\nu_{i}$ such that $X_{i}=E\left[Y_{i} \mid \mathcal{F}_{i}\right]$ for some $\sigma$-algebra $\mathcal{F}_{i} \subseteq \mathcal{A}_{i}$. Without loss of generality, we assume that the sets $\Omega_{i}$ are pairwise disjoint. We set $\Omega=\cup_{i=1}^{n} \Omega_{i}, \mathcal{A}=\sigma\left(\cup_{i=1}^{n} \mathcal{A}_{i}\right), P[A]=\sum_{i=1}^{n} p_{i} P_{i}\left[A \cap \Omega_{i}\right]$ for $A \in \mathcal{A}$ and $\mathcal{F}=\sigma\left(\cup_{i=1}^{n} \mathcal{F}_{i}\right)$. Furthermore, we define random variables $X=\sum_{i=1}^{n} X_{i} 1_{\Omega_{i}}$ and $Y=\sum_{i=1}^{n} Y_{i} 1_{\Omega_{i}}$ on $(\Omega, \mathcal{A}, P)$. Then, $\mathcal{L}_{X}=\sum_{i=1}^{n} p_{i} \mu_{i}, \mathcal{L}_{Y}=\sum_{i=1}^{n} p_{i} \nu_{i}$, and $E[Y \mid \mathcal{F}]=X$. Hence, (3.2) holds.

(d) The claim follows immediately from the conditional version of Jensen's inequality. 


\section{Comparison of ERRW on finite graphs with Polya urns}

Throughout this section, we consider linearly edge-reinforced random walk on a finite graph $G=(V, E)$ with starting point $\mathbf{0} \in V$ and initial weights $a=\left(a_{e}\right)_{e \in E}$. Let $X_{t}$, $t \in \mathbb{N}_{0}$, denote the location of the random walker at time $t$. Recall that we realize $X_{t}$ as the $t$-th projection defined on the subset $\Omega_{0} \subseteq V^{\mathbb{N}_{0}}$ of all admissible paths within the set $V^{\mathbb{N}_{0}}$ of all paths. Recall the definitions (1.1) and (1.3) of $w_{e}(t)$ and $w_{v}(t)$. For $v \in V$ and $t \in \mathbb{N}_{0}$, we abbreviate

$$
a_{v}=\sum_{\substack{e \ni v \\ e \in E}} a_{e}=w_{v}(0)
$$

Fix a vertex $v^{*} \in V$ and an edge $e^{*} \in E$ incident to $v^{*}$. For $n \in \mathbb{N}_{0}$, we define $T_{n}$, $n \in \mathbb{N}_{0}$, to denote the time of the $n+1$-st visit of the vertex $v^{*}$. Note that all these times are almost surely well-defined. Define the filtration $\mathcal{F}=\left(\mathcal{F}_{n}\right)_{n \in \mathbb{N}_{0}}$ with

$$
\mathcal{F}_{n}:=\sigma\left(X_{t}: 0 \leq t \leq T_{n}\right)
$$

encoding the observable information up to time $T_{n}$. We set

$$
M_{n}^{\mathrm{errw}}:=\frac{w_{e^{*}}\left(T_{n}\right)}{w_{v^{*}}\left(T_{n}\right)}=\frac{w_{e^{*}}\left(T_{n}\right)}{w_{v^{*}}\left(T_{0}\right)+2 n} .
$$

Here, we use that $w_{v^{*}}\left(T_{n}\right)=w_{v^{*}}\left(T_{0}\right)+2 n$, since between two subsequent visits of $v^{*}$, two (maybe coinciding) edges adjacent to $v^{*}$ have been crossed, one to leave $v^{*}$, and one to enter $v^{*}$ again. The random variable $M_{n}^{\text {errw }}$ equals the conditional probability given $\mathcal{F}_{n}$ that the edge-reinforced random walker leaves the vertex $v^{*}$ at time $T_{n}$ via the edge $e^{*}$.

Lemma 4.1 The sequence $\left(M_{n}^{\mathrm{errw}}\right)_{n \in \mathbb{N}_{0}}$ is a martingale with respect to $\mathcal{F}$.

Proof. By its definition (4.3), $M_{n}^{\text {errw }}$ is $\mathcal{F}_{n}$-measurable. Define a partition of $\Omega_{0}$ by

$$
\begin{aligned}
& A=\left\{\left\{X_{T_{n}}, X_{T_{n}+1}\right\}=e^{*},\left\{X_{T_{n+1}-1}, X_{T_{n+1}}\right\} \neq e^{*}\right\}, \\
& B=\left\{\left\{X_{T_{n}}, X_{T_{n}+1}\right\} \neq e^{*},\left\{X_{T_{n+1}-1}, X_{T_{n+1}}\right\}=e^{*}\right\}, \\
& C=\left\{\left\{X_{T_{n}}, X_{T_{n}+1}\right\}=e^{*},\left\{X_{T_{n+1}-1}, X_{T_{n+1}}\right\}=e^{*}\right\}, \\
& D=\left\{\left\{X_{T_{n}}, X_{T_{n}+1}\right\} \neq e^{*},\left\{X_{T_{n+1}-1}, X_{T_{n+1}}\right\} \neq e^{*}\right\} .
\end{aligned}
$$

The events $A, B, C, D$ distinguish whether the reinforced random walker leaves the vertex $v^{*}$ at time $T_{n}$ via $e^{*}$ or not and whether it enters $v^{*}$ at time $T_{n+1}$ via $e^{*}$ or not. We observe that

$$
w_{e^{*}}\left(T_{n+1}\right)= \begin{cases}w_{e^{*}}\left(T_{n}\right)+1 & \text { on } A \cup B, \\ w_{e^{*}}\left(T_{n}\right)+2 & \text { on } C, \\ w_{e^{*}}\left(T_{n}\right) & \text { on } D\end{cases}
$$


Let us consider the transformation $\Pi_{n+1}: \Omega_{0} \rightarrow \Omega_{0}$ which reverts the orientation of the $n+1$-st excursion from $v^{*}$, i.e. the excursion between time $T_{n}$ and time $T_{n+1}$, while keeping the rest of the path unchanged. By partial exchangeability (see e.g. Lemma 2 of [KR00]), this transformation is measure preserving. Furthermore, it maps $A$ bijectively to $B$. Hence, abbreviating $P:=P_{\mathbf{0}, a}^{G}$, the formula $P\left[A \mid \mathcal{F}_{n}\right]=P\left[B \mid \mathcal{F}_{n}\right]$ is valid. Note that

$$
\begin{aligned}
& P\left[A \mid \mathcal{F}_{n}\right]+P\left[C \mid \mathcal{F}_{n}\right]=M_{n}^{\text {errw }} \text { and } \\
& P\left[B \mid \mathcal{F}_{n}\right]+P\left[D \mid \mathcal{F}_{n}\right]=1-M_{n}^{\text {errw }}
\end{aligned}
$$

hold. Consequently,

$$
\begin{aligned}
E\left[M_{n+1}^{\text {errw }} \mid \mathcal{F}_{n}\right]= & \frac{w_{e^{*}}\left(T_{n}\right)+1}{w_{v^{*}}\left(T_{0}\right)+2 n+2}\left(P\left[A \mid \mathcal{F}_{n}\right]+P\left[B \mid \mathcal{F}_{n}\right]\right)+\frac{w_{e^{*}}\left(T_{n}\right)+2}{w_{v^{*}}\left(T_{0}\right)+2 n+2} P\left[C \mid \mathcal{F}_{n}\right] \\
& +\frac{w_{e^{*}}\left(T_{n}\right)}{w_{v^{*}}\left(T_{0}\right)+2 n+2} P\left[D \mid \mathcal{F}_{n}\right] \\
= & \frac{w_{e^{*}}\left(T_{n}\right)+1}{w_{v^{*}}\left(T_{0}\right)+2 n+2} \cdot 2 P\left[A \mid \mathcal{F}_{n}\right]+\frac{w_{e^{*}}\left(T_{n}\right)+2}{w_{v^{*}}\left(T_{0}\right)+2 n+2}\left(M_{n}^{\text {errw }}-P\left[A \mid \mathcal{F}_{n}\right]\right) \\
& +\frac{w_{e^{*}}\left(T_{n}\right)}{w_{v^{*}}\left(T_{0}\right)+2 n+2}\left(1-M_{n}^{\text {errw }}-P\left[A \mid \mathcal{F}_{n}\right]\right) \\
= & M_{n}^{\text {errw }} .
\end{aligned}
$$

Hence, $\left(M_{n}^{\text {errw }}\right)_{n \in \mathbb{N}_{0}}$ is a martingale.

Now consider the star subgraph $G^{\text {polya }}=\left(V^{\text {polya }}, E^{\text {polya }}\right)$ of $G$ consisting of $v^{*}$ and its immediate neighbors and the edges connecting $v^{*}$ with its immediate neighbors. We consider edge-reinforced random walk $\left(X_{t}^{\text {polya }}\right)_{t \in \mathbb{N}_{0}}$ on $G^{\text {polya }}$ starting in $v^{*}$ with random initial weights $\left(a_{e}^{\text {polya }}\right)_{e \in E^{\text {polya }}}$ having the same joint distribution as $\left(w_{e}\left(T_{0}\right)\right)_{e \in E^{\text {polya }}}$, the weights of the edges $e \in E^{\text {polya }}$ for the edge-reinforced random walk on $G$ at time $T_{0}$. Let us explain the difference between $a_{e}$ and $a_{e}^{\text {polya }}$ : If $v^{*}=\mathbf{0}$, then $\left(a_{e}^{\text {polya }}\right)_{e \in E^{\text {polya }}}$ just equals $\left(a_{e}\right)_{e \in E^{\text {polya }}}$. But if $v^{*} \neq \mathbf{0}$, then $v^{*}$ is entered the first time via a random edge $\tilde{e}=\left\{X_{T_{0}-1}, X_{T_{0}}\right\} \in E^{\text {polya }}$; then one has $w_{\tilde{e}}\left(T_{0}\right)=a_{\tilde{e}}+1$, while $w_{e}\left(T_{0}\right)=a_{e}$ for all other edges $e \in E^{\text {polya }} \backslash\{\tilde{e}\}$.

For this reinforced random walk on the subgraph $G^{\text {polya }}$, we introduce similar notation as for the reinforced random walk on the full graph $G$ : Let $w_{e}^{\text {polya }}(t), e \in E^{\text {polya }}$, denote the weight of the edge $e$ at time $t$, and $T_{n}^{\text {polya }}=2 n$ is the time of the $n+1$-st visit of the vertex $v^{*}$. We set

$$
\begin{aligned}
& a_{v^{*}}^{\text {polya }}=\sum_{e \in E_{\text {polya }}} a_{e}^{\text {polya }}=\left\{\begin{array}{l}
a_{v^{*}} \text { if } \mathbf{0}=v^{*}, \\
a_{v^{*}}+1 \text { if } \mathbf{0} \neq v^{*},
\end{array}\right. \\
& w_{v^{*}}^{\text {polya }}(t)=\sum_{e \in E^{\text {polya }}} w_{e}^{\text {polya }}(t), \\
& M_{n}^{\text {polya }}:=\frac{w_{e^{*}}^{\text {polya }}\left(T_{n}^{\text {polya }}\right)}{w_{v^{*}}^{\text {polya }}\left(T_{n}^{\text {polya }}\right)}=\frac{w_{e^{*}}^{\text {polya }}(2 n)}{a_{v^{*}}^{\text {polya }}+2 n} .
\end{aligned}
$$


Note that the denominator $a_{v^{*}}^{\text {polya }}+2 n$ is not random.

Remark 4.2 In down-to-earth terms, $\left(M_{n}^{\text {polya }}\right)_{n \in \mathbb{N}_{0}}$ is a Polya urn model, at least if the initial weights are natural numbers: Consider an urn containing initially $a_{e^{*}}^{\text {polya }}$ red and $a_{v^{*}}^{\text {polya }}-a_{e^{*}}^{\text {polya }}$ blue balls. In each discrete time step, draw a ball at random and put it back together with two balls of the same color. Then, the probability to draw a red ball in the $n+1$-st drawing equals $M_{n}^{\text {polya }}$. It is well-known that $\left(M_{n}^{\text {polya }}\right)_{n \in \mathbb{N}_{0}}$ is a martingale.

Furthermore, $\left(M_{n}^{\text {polya }}\right)_{n \in \mathbb{N}_{0}}$ is a time-inhomogeneous Markov chain with random initial state $M_{0}^{\text {polya }}=a_{e^{*}}^{\text {polya }} / a_{v^{*}}^{\text {polya }}$ and transitions

$$
M_{n+1}^{\text {polya }}= \begin{cases}\alpha_{n} M_{n}^{\text {polya }}+1-\alpha_{n} & \text { with probability } M_{n}^{\text {polya }}, \\ \alpha_{n} M_{n}^{\text {polya }} & \text { with probability } 1-M_{n}^{\text {polya }},\end{cases}
$$

where we have set

$$
\alpha_{n}=\frac{a_{v^{*}}^{\text {polya }}+2 n}{a_{v^{*}}^{\text {polya }}+2 n+2} .
$$

The following lemma plays a central role in the whole article: It compares reinforced random walk on any finite graph with a much simpler Polya urn model.

Lemma 4.3 For all $n \in \mathbb{N}$, one has $M_{n}^{\text {errw }} \triangleleft M_{n}^{\text {polya }}$.

Proof. The proof is by induction. The claim is obvious for $n=0$, since $M_{0}^{\text {errw }}$ has the same distribution as $M_{0}^{\text {polya }}$. For the step $n \leadsto n+1$, we introduce the Polya urn transition kernel

$$
K_{n}:[0,1] \times \mathcal{B}([0,1]) \rightarrow[0,1], \quad K_{n}(x, \cdot):=x \delta_{\alpha_{n} x+1-\alpha_{n}}+(1-x) \delta_{\alpha_{n} x},
$$

which was already described in (4.15). We define an auxiliary random variable $M_{n+1}^{\text {aux }}$ by

$$
M_{n}^{\text {aux }}= \begin{cases}\alpha_{n} M_{n}^{\text {errw }}+1-\alpha_{n} & \text { for }\left\{X_{T_{n}}, X_{T_{n}+1}\right\}=e^{*} \\ \alpha_{n} M_{n}^{\text {errw }} & \text { otherwise. }\end{cases}
$$

It has the law

$$
\mathcal{L}_{M_{n}^{\text {aux }}}=\mathcal{L}_{M_{n}^{\text {errw }}} K_{n}
$$

For an interpretation of $M_{n}^{\text {aux }}$ in the case of integer weights, consider an urn containing $w_{e^{*}}\left(T_{n}\right)$ red and $w_{v^{*}}\left(T_{n}\right)-w_{e^{*}}\left(T_{n}\right)$ blue balls. Draw a ball at random and put it back together with two balls of the same color. Then, the probability to draw a red ball in the next drawing equals $M_{n}^{\text {aux }}$.

We claim that

$$
M_{n+1}^{\text {errw }} \triangleleft M_{n+1}^{\text {aux }} \quad \text { and } \quad M_{n+1}^{\text {aux }} \triangleleft M_{n+1}^{\text {polya }}
$$

hold. Since the relation $\triangleleft$ is transitive by Lemma 3.2 , these claims imply our goal $M_{n+1}^{\text {errw }} \triangleleft$ $M_{n+1}^{\text {polya }}$. 
To prove the first claim $M_{n+1}^{\text {errw }} \triangleleft M_{n+1}^{\text {aux }}$, we introduce the following $\sigma$-field:

$$
\mathcal{G}_{n+1}=\sigma\left(w_{e}\left(T_{k}\right): e \in E ; k=0, \ldots, n+1\right) .
$$

(Note that $\mathcal{G}_{n+1}$ contains less information than $\mathcal{F}_{n+1}$. In particular, the orientation of the $n+1$ st excursion from $v^{*}$ of the random walker is not measurable with respect to $\mathcal{G}_{n+1}$.)

We now show that

$$
M_{n+1}^{\mathrm{errw}}=E\left[M_{n+1}^{\mathrm{aux}} \mid \mathcal{G}_{n+1}\right]
$$

holds, which implies the first claim $M_{n+1}^{\text {errw }} \triangleleft M_{n+1}^{\text {aux }}$. Indeed, $M_{n+1}^{\text {errw }}$ is $\mathcal{G}_{n+1}$-measurable, and $M_{n+1}^{\text {errw }}=M_{n+1}^{\text {aux }}$ holds on the two $\mathcal{G}_{n+1}$-measurable events $\left\{w_{e^{*}}\left(T_{n+1}\right)=w_{e^{*}}\left(T_{n}\right)\right\}$ and $\left\{w_{e^{*}}\left(T_{n+1}\right)=w_{e^{*}}\left(T_{n}\right)+2\right\}$. The complement of the union of these two events equals

$$
\left\{w_{e^{*}}\left(T_{n+1}\right)=w_{e^{*}}\left(T_{n}\right)+1\right\}=A \cup B,
$$

with the two disjoint events $A$ and $B$ defined in (4.4) and (4.5). Note that $M_{n+1}^{\text {aux }}$ equals $\alpha_{n} M_{n}^{\text {errw }}+1-\alpha_{n}$ on $A$ and $\alpha_{n} M_{n}^{\text {errw }}$ on $B$. Consider again the transformation $\Pi_{n+1}$ : $\Omega_{0} \rightarrow \Omega_{0}$ which reverts the orientation of the excursion between time $T_{n}$ and time $T_{n+1}$, while keeping the rest of the path unchanged; $\Pi_{n+1}$ was introduced after (4.8). For every random variable $X$ taking values in $[0,1]$, one has $E\left[X \circ \Pi_{n+1} \mid \mathcal{G}_{n+1}\right]=E\left[X \mid \mathcal{G}_{n+1}\right]$, i.e. $\mathcal{G}_{n+1}$ cannot distinguish between any path $\omega \in \Omega_{\mathbf{0}}$ and its partially time-reversed variant $\Pi_{n+1}(\omega)$. Furthermore,

$$
\frac{1}{2}\left(M_{n+1}^{\text {aux }}+M_{n+1}^{\text {aux }} \circ \Pi_{n+1}\right)=M_{n+1}^{\text {errw }}
$$

holds true. Using that $M_{n+1}^{\text {errw }}$ is measurable with respect to $\mathcal{G}_{n+1}$, we conclude:

$$
E\left[M_{n+1}^{\mathrm{aux}} \mid \mathcal{G}_{n+1}\right] 1_{A \cup B}=\frac{1}{2} E\left[M_{n+1}^{\mathrm{aux}}+M_{n+1}^{\mathrm{aux}} \circ \prod_{n+1} \mid \mathcal{G}_{n+1}\right] 1_{A \cup B}=M_{n+1}^{\mathrm{errw}} 1_{A \cup B},
$$

which finishes the proof of the claim (4.22).

The second claim $M_{n+1}^{\text {aux }} \triangleleft M_{n+1}^{\text {polya }}$ in $(4.20)$, i.e. $\mathcal{L}_{M_{n}^{\text {errw }}} K_{n} \triangleleft \mathcal{L}_{M_{n}^{\text {polya }}} K_{n}$, is a consequence of the induction hypothesis $M_{n}^{\text {errw }} \triangleleft M_{n}^{\text {polya }}$ and the following lemma.

Lemma 4.4 If $\mu$ is a discrete distribution on $[0,1]$ and $\nu$ is any distribution on $[0,1]$ with $\mu \triangleleft \nu$, then $\mu K_{n} \triangleleft \nu K_{n}$ also holds.

Proof. We prove the lemma first in the special case $\mu=\delta_{x}, x \in[0,1]$. Thus we claim: If the distribution $\nu$ has the expectation $x$, then one has

$$
x \delta_{\alpha_{n} x+1-\alpha_{n}}+(1-x) \delta_{\alpha_{n} x} \triangleleft \nu K_{n} .
$$

To prove this, let $X$ and $Y$ be [0,1]-valued random variables with joint distribution $\nu \otimes K_{n}$, i.e. with joint distribution $\nu(d x) K_{n}(x, d y)$. We abbreviate the variance of $\nu$ by $\sigma^{2}$. Note 
that $Y \geq X$ is the same as $Y=\alpha_{n} X+1-\alpha_{n}$ and $Y<X$ is the same as $Y=\alpha_{n} X$ a.s. We get:

$$
\begin{aligned}
& E[Y, Y \geq X]=\int_{[0,1]}\left(\alpha_{n} y+1-\alpha_{n}\right) y \nu(d y)=\left(\alpha_{n} x+1-\alpha_{n}\right) x+\alpha_{n} \sigma^{2}, \\
& P[Y \geq X]=\int_{[0,1]} y \nu(d y)=x, \\
& E[Y, Y<X]=\int_{[0,1]} \alpha_{n} y(1-y) \nu(d y)=\alpha_{n} x(1-x)-\alpha_{n} \sigma^{2}, \\
& P[Y<X]=\int_{[0,1]}(1-y) \nu(d y)=1-x .
\end{aligned}
$$

Thus,

$$
x \delta_{\alpha_{n} x+1-\alpha_{n}+\alpha_{n} \sigma^{2} / x}+(1-x) \delta_{\alpha_{n} x-\alpha_{n} \sigma^{2} /(1-x)} \sim E[Y \mid \sigma(\{Y \geq X\})] \triangleleft Y \sim \nu K_{n} .
$$

Finally, we show that

$$
x \delta_{\alpha_{n} x+1-\alpha_{n}}+(1-x) \delta_{\alpha_{n} x} \triangleleft x \delta_{\alpha_{n} x+1-\alpha_{n}+\alpha_{n} \sigma^{2} / x}+(1-x) \delta_{\alpha_{n} x-\alpha_{n} \sigma^{2} /(1-x)} .
$$

Since $\triangleleft$ is transitive, (4.31) and (4.32) imply the claim (4.26).

To verify (4.32), we show more generally that for any numbers $A \geq C \geq D \geq B$ with $x A+(1-x) B=x C+(1-x) D$, the relation

$$
x \delta_{C}+(1-x) \delta_{D} \triangleleft x \delta_{A}+(1-x) \delta_{B}
$$

holds. We apply this then with

$$
A=\alpha_{n} x+1-\alpha_{n}+\alpha_{n} \frac{\sigma^{2}}{x} \geq C=\alpha_{n} x+1-\alpha_{n} \geq D=\alpha_{n} x \geq B=\alpha_{n} x-\alpha_{n} \frac{\sigma^{2}}{1-x} .
$$

Before proving (4.33) formally, let us interpret it intuitively: Suppose we have two bottles of vinegar, having volumes $x$ and $1-x$ and concentrations $A$ and $B$, respectively. By partially mixing the content of the bottles, we can obtain two other bottles of vinegar, also having volumes $x$ and $1-x$, but concentrations $C$ and $D$, respectively.

For $A=B$, there is nothing to show. Otherwise, take $\Omega=\{C, D\} \times\{A, B\}$ with the probability measure

$$
\frac{x(C-B)}{A-B} \delta_{(C, A)}+\frac{(1-x)}{A-B}\left\{(D-B)\left[\delta_{(C, B)}+\delta_{(D, A)}\right]+(A-D) \delta_{(D, B)}\right\},
$$

and let $\omega_{1}$ and $\omega_{2}$ denote the projections on the first and second coordinate, respectively. Then, $E\left[\omega_{2} \mid \omega_{1}\right]=\omega_{1} \sim x \delta_{C}+(1-x) \delta_{D}$ and $\omega_{2} \sim x \delta_{A}+(1-x) \delta_{B}$. 
To prove the lemma in the general case, we write

$$
\mu=\sum_{i=1}^{n} p_{i} \delta_{x_{i}}
$$

Let $X \sim \mu$ and $Y \sim \nu$ be random variables with $X=E[Y \mid X]$. For $i=1, \ldots, n$, we define

$$
\nu_{i}=P\left[Y \in \cdot X=x_{i}\right]
$$

to denote the conditional distribution of $Y$ given $X=x_{i}$. Then $\delta_{x_{i}} \triangleleft \nu_{i}$. By the already proven special case, we infer

$$
\delta_{x_{i}} K_{n} \triangleleft \nu_{i} K_{n}
$$

The claim

$$
\mu K_{n}=\sum_{i=1}^{n} p_{i} \delta_{x_{i}} K_{n} \triangleleft \sum_{i=1}^{n} p_{i} \nu_{i} K_{n}=\nu K_{n}
$$

now follows from Lemma 3.2(c).

Let $\beta(a, b)$ denote the beta distribution with parameters $a, b>0$.

Lemma 4.5 As $n \rightarrow \infty$, the sequence $\left(M_{n}^{\text {polya }}\right)_{n \in \mathbb{N}}$ converges almost surely to a random limit with distribution

$$
\begin{aligned}
& \mathbb{Q}^{\text {polya }}=\mathbb{Q}_{0, a, v^{*}, e^{*}}^{\text {polya } G} \\
& = \begin{cases}\beta\left(\frac{a_{e^{*}}}{2}, \frac{a_{v^{*}}-a_{e^{*}}}{2}\right) & \text { if } v^{*}=\mathbf{0}, \\
P_{\mathbf{0}, a}^{G}\left[w_{e^{*}}\left(T_{0}\right)=a_{e^{*}}+1\right] \beta\left(\frac{a_{e^{*}}+1}{2}, \frac{a_{v^{*}}-a_{e^{*}}}{2}\right) & \\
+P_{\mathbf{0}, a}^{G}\left[w_{e^{*}}\left(T_{0}\right)=a_{e^{*}}\right] \beta\left(\frac{a_{e^{*}}}{2}, \frac{a_{v^{*}}-a_{e^{*}}+1}{2}\right) & \text { if } v^{*} \neq \mathbf{0} .\end{cases}
\end{aligned}
$$

Proof. Recall Remark 4.2. To make the proof more intuitive, we use the language of the Polya urn model, by abuse of notation also in the case where the initial weights are not natural numbers. We consider an urn with a random initial composition consisting of $a_{e^{*}}^{\text {polya }}$ red and $a_{v^{*}}^{\text {polya }}-a_{e^{*}}^{\text {polya }}$ blue balls. The dynamics consists of drawing a ball at random from the urn and putting it back into the urn together with two balls of the same color. Conditioned on the initial number of balls $a_{e^{*}}^{\text {polya }}$ and $a_{v^{*}}^{\text {polya }}-a_{e^{*}}^{\text {polya }}$, the fraction of red balls in the urn after $n$ drawings, namely $M_{n}^{\text {polya }}$, converges almost surely to a beta distribution with parameters $a_{e^{*}}^{\text {polya }} / 2$ and $\left(a_{v^{*}}^{\text {polya }}-a_{e^{*}}^{\text {polya }}\right) / 2$; see e.g. [Dur04], Section $4.3 \mathrm{~b}$, page 238. The initial composition is distributed according to

$$
\begin{aligned}
& \left(a_{e^{*}}^{\text {polya }}, a_{v^{*}}^{\text {polya }}-a_{e^{*}}^{\text {polya }}\right) \\
= & \begin{cases}\left(a_{e^{*}}, a_{v^{*}}-a_{e^{*}}\right) & \text { if } v^{*}=\mathbf{0}, \\
\left(a_{e^{*}}+1, a_{v^{*}}-a_{e^{*}}\right) & \text { with probability } P_{\mathbf{0}, a}^{G}\left[w_{e^{*}}\left(T_{0}\right)=a_{e^{*}}+1\right] \text { if } v^{*} \neq \mathbf{0}, \\
\left(a_{e^{*}}, a_{v^{*}}-a_{e^{*}}+1\right) & \text { with probability } P_{\mathbf{0}, a}^{G}\left[w_{e^{*}}\left(T_{0}\right)=a_{e^{*}}\right] \text { if } v^{*} \neq \mathbf{0} .\end{cases}
\end{aligned}
$$


The claim follows.

It is well-known (see e.g. Theorem 3.1 of [Rol03]) that the edge-reinforced random walk on a finite graph $G$ has the same distribution as a random walk in a random environment given by random weights on the edges. Let

$$
\mathbb{Q}^{\text {errw }}=\mathbb{Q}_{0, a}^{G}
$$

denote the unique mixing measure on the simplex $\Delta:=\left\{\left(x_{e}\right)_{e \in E} \in(0,1)^{E}: \sum_{e \in E} x_{e}=1\right\}$.

Theorem 4.6 For any finite graph $G$, any starting point $\mathbf{0}$, any initial weights a, any vertex $v^{*}$, and any edge $e^{*}$ incident to $v^{*}$, the distribution $\mathbb{Q}_{0, a}^{G}\left[x_{e^{*}} / x_{v^{*}} \in \cdot\right]$ of $x_{e^{*}} / x_{v^{*}}$ with respect to $\mathbb{Q}_{\mathbf{0}, a}^{G}$ fulfills

$$
\mathbb{Q}_{\mathbf{0}, a}^{G}\left[\frac{x_{e^{*}}}{x_{v^{*}}} \in \cdot\right] \triangleleft \mathbb{Q}_{\mathbf{0}, a, v^{*}, e^{*}}^{\mathrm{polya}, G}
$$

Proof. Recall the abbreviations (4.40) and (4.42). Both, $\left(M_{n}^{\text {errw }}\right)_{n \in \mathbb{N}}$ and $\left(M_{n}^{\text {polya }}\right)_{n \in \mathbb{N}}$ are bounded and hence uniformly integrable martingales. By Lemma 4.5, the limit of $\left(M_{n}^{\text {polya }}\right)_{n \in \mathbb{N}}$ has the distribution $\mathbb{Q}^{\text {polya }}$. Since the edge-reinforced random walk on the finite graph $G$ is a mixture of recurrent Markov chains, we know that $\left(w_{t}(e) / t\right)_{e \in E}$ converges almost surely as $t \rightarrow \infty$ to a limit with distribution $\mathbb{Q}^{\text {errw }}$. Consequently, $M_{n}^{\text {errw }}=w_{e^{*}}\left(T_{n}\right) / w_{v^{*}}\left(T_{n}\right)$ converges almost surely as $n \rightarrow \infty$ to a random limit with distribution $\mathbb{Q}^{\text {errw }}\left[x_{e^{*}} / x_{v^{*}} \in \cdot\right]$. Using that $M_{n}^{\text {errw }} \triangleleft M_{n}^{\text {polya }}$ for all $n$ by Lemma 4.3 , the claim follows from Lemma $3.2(\mathrm{~b})$.

\section{Proofs of the main results}

First, we prove the following uniform tail estimates for $x_{e} / x_{v}$ :

Lemma 5.1 Theorem 2.3 holds for all finite graphs $G$.

Proof. Recall the abbreviation (4.42). Since $\mathbb{Q}^{\text {errw }}\left[x_{e} / x_{v} \leq \varepsilon\right] \leq 1$ and $\mathbb{Q}^{\text {errw }}\left[x_{e} / x_{v} \geq\right.$ $1-\varepsilon] \leq 1$, it suffices to prove the estimates $(2.3)$ for all $\varepsilon \in(0,1 / 3)$. The claim follows then with possibly larger constants $c_{1}$ and $c_{2}$.

Let $G$ be a finite graph, let $\mathbf{0}$ be any vertex, and let $a \in \mathbb{R}_{+}^{E}$ be any initial weights. In this case, it is known that the measure $\mathbb{Q}^{\text {errw }}$ satisfies the assertions of Theorem 2.2 (see e.g. Theorem 3.1 of [Rol03]).

Let $v \in V$ and let $e \in E$ be an edge incident to $v$. If degree $(v)=1$, then $x_{e} / x_{v}=1$ and $a_{v}=a_{e}$. Hence, the left estimate in (2.3) always holds and the right estimate in (2.3) holds for any $c_{2} \geq 1$. In the following, we assume degree $(v) \geq 2$. Combining Theorem 4.6 with Lemma 3.2(d) yields for any convex bounded function $f:[0,1] \rightarrow \mathbb{R}$ the inequality

$$
E_{\mathbb{Q}^{\text {errw }}}\left[f\left(\frac{x_{e}}{x_{v}}\right)\right] \leq \int_{0}^{1} f(x) \mathbb{Q}^{\text {polya }}(d x) .
$$


Let $\varepsilon \in(0,1 / 3)$. We apply the last inequality with

$$
f(x)= \begin{cases}1-\frac{x}{2 \varepsilon} & \text { for } 0 \leq x \leq 2 \varepsilon \\ 0 & \text { for } 2 \varepsilon \leq x \leq 1\end{cases}
$$

to obtain

$$
\begin{aligned}
\frac{1}{2} \mathbb{Q}^{\text {errw }}\left[\frac{x_{e}}{x_{v}} \leq \varepsilon\right] & \leq E_{\mathbb{Q}^{\text {errw }}}\left[f\left(\frac{x_{e}}{x_{v}}\right)\right] \\
& \leq \int_{0}^{1} f(x) \mathbb{Q}^{\text {polya }}(d x) \leq \mathbb{Q}^{\text {polya }}[[0,2 \varepsilon]]
\end{aligned}
$$

Recall the definition $(4.40)$ of $\mathbb{Q}^{\text {polya }}$. In the case $v \neq \mathbf{0}$, we first estimate

$$
I_{1}:=P_{\mathbf{0}, a}^{G}\left[w_{e}\left(T_{0}\right)=a_{e}+1\right] \cdot \frac{1}{\mathrm{~B}\left(\frac{a_{e}+1}{2}, \frac{a_{v}-a_{e}}{2}\right)} \int_{0}^{2 \varepsilon} x^{\left(a_{e}+1\right) / 2-1}(1-x)^{\left(a_{v}-a_{e}\right) / 2-1} d x,
$$

where B denotes the Beta function. Clearly, $P_{\mathbf{0}, a}^{G}\left[w_{e}\left(T_{0}\right)=a_{e}+1\right] \leq 1$. Since the Beta function is continuous, the normalizing constant of the beta distribution in (5.4) depends continuously on $a_{v}$ and $a_{e}$. To bound the last integral, we use $1 / 3 \leq 1-2 \varepsilon \leq 1-x \leq 1$ for all $x \in(0,2 \varepsilon)$. This yields the estimate

$$
I_{1} \leq c_{4}\left(a_{v}, a_{e}\right) \varepsilon^{\left(a_{e}+1\right) / 2} \leq c_{4}\left(a_{v}, a_{e}\right) \varepsilon^{a_{e} / 2}
$$

with a constant $c_{4}\left(a_{v}, a_{e}\right)>0$ depending continuously on $a_{v}$ and $a_{e}$ and not depending on any other quantity, in particular, not depending on $G, \mathbf{0}, v$ and $e$. An analogous argument yields

$$
\begin{aligned}
I_{2} & :=P_{\mathbf{0}, a}^{G}\left[w_{e}\left(T_{0}\right)=a_{e}\right] \cdot \frac{1}{\mathrm{~B}\left(\frac{a_{e}}{2}, \frac{a_{v}-a_{e}+1}{2}\right)} \int_{0}^{2 \varepsilon} x^{a_{e} / 2-1}(1-x)^{\left(a_{v}-a_{e}+1\right) / 2-1} d x \\
& \leq c_{5}\left(a_{v}, a_{e}\right) \varepsilon^{a_{e} / 2}
\end{aligned}
$$

with a constant $c_{5}\left(a_{v}, a_{e}\right)>0$ depending continuously on $a_{e}$ and $a_{v}$. Combining (5.5) and (5.6), we conclude

$$
\mathbb{Q}^{\text {polya }}[[0,2 \varepsilon]] \leq\left[c_{4}\left(a_{v}, a_{e}\right)+c_{5}\left(a_{v}, a_{e}\right)\right] \varepsilon^{a_{e} / 2}
$$

In the case $v=\mathbf{0}$, a similar argument yields the bound (5.7) with a different constant. This proves the estimates for the lower tail probabilities in Theorem 2.3.

To bound the upper tail probabilities, we apply (5.1) with

$$
f(x)= \begin{cases}0 & \text { for } 0 \leq x \leq 1-2 \varepsilon \\ 1-\frac{1-x}{2 \varepsilon} & \text { for } 1-2 \varepsilon \leq x \leq 1\end{cases}
$$


This yields

$$
\begin{aligned}
\frac{1}{2} \mathbb{Q}^{\text {errw }}\left[\frac{x_{e}}{x_{v}} \geq 1-\varepsilon\right] & \leq E_{\mathbb{Q}^{\text {errw }}}\left[f\left(\frac{x_{e}}{x_{v}}\right)\right] \\
& \leq \int_{0}^{1} f(x) \mathbb{Q}^{\text {polya }}(d x) \leq \mathbb{Q}^{\text {polya }}[[1-2 \varepsilon, 1]] .
\end{aligned}
$$

We indicate only the estimate in the case $v=\mathbf{0}$. In this case, one gets

$$
\begin{aligned}
\mathbb{Q}^{\text {polya }}[[1-2 \varepsilon, 1]] & =\frac{1}{\mathrm{~B}\left(\frac{a_{e}}{2}, \frac{a_{v}-a_{e}}{2}\right)} \int_{1-2 \varepsilon}^{1} x^{a_{e} / 2-1}(1-x)^{\left(a_{v}-a_{e}\right) / 2-1} d x \\
& =\frac{1}{\mathrm{~B}\left(\frac{a_{e}}{2}, \frac{a_{v}-a_{e}}{2}\right)} \int_{0}^{2 \varepsilon} x^{\left(a_{v}-a_{e}\right) / 2-1}(1-x)^{a_{e} / 2-1} d x
\end{aligned}
$$

Again, similar arguments as above imply

$$
\mathbb{Q}^{\text {polya }}[[1-2 \varepsilon, 1]] \leq c_{6}\left(a_{v}, a_{e}\right) \varepsilon^{\left(a_{v}-a_{e}\right) / 2}
$$

with a constant $c_{6}\left(a_{v}, a_{e}\right)>0$ depending continuously on $a_{v}$ and $a_{e}$.

Lemma 5.2 Theorem 2.4 holds for all finite graphs $G$.

Proof. Given a compact set $K \subset(0, \infty)$, we set

$$
c_{3}(K)=\sup _{\substack{a, a^{\prime} \in K \\ a \geq a^{\prime}}} c_{1}\left(a, a^{\prime}\right)<\infty,
$$

where $c_{1}\left(a, a^{\prime}\right)$ is taken from Lemma 5.1, the version of Theorem 2.3 for finite graphs. Now take a finite graph $G=(V, E)$, a starting point $\mathbf{0} \in V$, and initial weights $a \in \mathbb{R}_{+}^{E}$. By Lemma 5.1, $\mathbb{Q}^{\text {errw }}$ has the properties specified in Theorems 2.2 and 2.3. Given any edges $e, f \in E$, any path

$$
\stackrel{e=e_{0}}{\longrightarrow} v_{1} \stackrel{e_{1}}{\longrightarrow} v_{2} \stackrel{e_{2}}{\longrightarrow} \ldots \stackrel{e_{l-1}}{\longrightarrow} v_{l} \stackrel{e_{l}=f}{\longrightarrow}
$$

from $e$ to $f$, any $M>0$, and assuming that the initial weights $\left(a_{e^{\prime}}\right)_{e^{\prime} \in E}$ satisfy $a_{e_{i}} \in K$ and $a_{v_{j}}=\sum_{e^{\prime} \ni v_{j}} a_{e^{\prime}} \in K(i=0, \ldots, l ; j=1, \ldots, l)$, we estimate, using Lemma 5.1 in the last but one step:

$$
\begin{aligned}
& \mathbb{Q}^{\text {errw }}\left[x_{e} \geq M x_{f}\right] \leq \mathbb{Q}^{\text {errw }}\left[x_{e_{i-1}} \geq M^{1 / l} x_{e_{i}} \text { for at least one } i=1, \ldots, l\right] \\
& \leq \sum_{i=1}^{l} \mathbb{Q}^{\text {errw }}\left[x_{e_{i-1}} \geq M^{1 / l} x_{e_{i}}\right] \leq \sum_{i=1}^{l} \mathbb{Q}^{\text {errw }}\left[x_{v_{i}} \geq M^{1 / l} x_{e_{i}}\right] \\
& \leq \sum_{i=1}^{l} c_{1}\left(a_{v_{i}}, a_{e_{i}}\right) M^{-a_{e_{i}} / 2 l} \leq c_{3}(K) l M^{-\gamma}
\end{aligned}
$$


where $\gamma$ is defined in (2.6).

Let $G=(V, E)$ be a locally finite graph, and let $G_{n}=\left(V_{n}, E_{n}\right)$ be finite connected subgraphs of $G$ with $V_{n} \uparrow V$ and $E_{n} \uparrow E$, and $\mathbf{0} \in V_{n}$ for all $n$. We denote by $\mathbb{Q}_{n}^{\text {errw }}:=\mathbb{Q}_{0, a}^{G_{n}}$ the unique mixing measure as defined in (4.42) for the edgereinforced random walk on the graph $G_{n}$. By its definition, $\mathbb{Q}_{n}^{\text {errw }}$ is a measure on the simplex $\Delta_{n}=\left\{\left(x_{e}\right)_{e \in E_{n}} \in(0,1)^{E_{n}}: \sum_{e \in E_{n}} x_{e}=1\right\}$. We introduce the canonical projections $x_{e}: \Delta_{n} \rightarrow(0,1), e \in E_{n}$, suppressing the dependence on $n$ in the notation.

Fix $e_{0} \in E$. We assume without loss of generality that $e_{0} \in E_{n}$ for all $n$. We set $\tilde{x}_{e}=x_{e} / x_{e_{0}}$ for $e \in E_{n}$. In particular, $\tilde{x}_{e_{0}}=1$ holds. The weights $\left(x_{e}\right)_{e \in E_{n}}$ and $\left(\tilde{x}_{e}\right)_{e \in E_{n}}$ are multiples of each other; hence they induce the same Markov chain $\left(Q_{\mathbf{0}, x}^{G_{n}}=Q_{\mathbf{0}}^{G_{n}}\right)$. Thus, we can use the law of $\left(\tilde{x}_{e}\right)_{e \in E_{n}}$ with respect to $\mathbb{Q}_{n}^{\text {errw }}$ as mixing measure for the edge-reinforced random walk on $G_{n}$.

Lemma 5.3 There exists a strictly increasing sequence $(n(k))_{k \in \mathbb{N}}$ in $\mathbb{N}$ such that for all finite $F \subseteq E$, the law of $\left(\tilde{x}_{e}\right)_{e \in F}$ with respect to $\mathbb{Q}_{n(k)}^{\text {err }}$ converges weakly as $k \rightarrow \infty$ to a distribution, supported on $(0, \infty)^{F}$.

Proof. We prove first that for any fixed $k \in \mathbb{N}$, there exists $n_{0}=n_{0}(k) \geq k$ such that the distributions $\mathbb{Q}_{n}^{\text {errw }}\left[\left(\ln \tilde{x}_{e}\right)_{e \in E_{k}} \in \cdot\right], n \geq n_{0}$, are tight. Fix $e \in E_{k}$ and a path $\pi$ :

$$
\stackrel{e_{0}}{\longrightarrow} v_{1} \stackrel{e_{1}}{\longrightarrow} v_{2} \stackrel{e_{2}}{\longrightarrow} \ldots \stackrel{e_{l-1}}{\longrightarrow} v_{l} \stackrel{e_{l}=e}{\longrightarrow}
$$

from $e_{0}$ to $e$ in $E_{k}$. Choose $n_{0} \in \mathbb{N}$ so large that for any $v_{j}$ in the path $\pi$, any edge $e \in E$ incident to $v_{j}$ belongs to $E_{n_{0}}$. Let $K:=\left\{a_{e_{i}}, a_{v_{j}}: i=0, \ldots, l, j=1, \ldots, l\right\}$ and let $\gamma$ be as in (2.6). Then, by Lemma 5.2 (the finite graph version of Theorem 2.4), taking $c_{3}(K)$ as in that lemma, we obtain for all $n \geq n_{0}$ and all $M>0$ :

$$
\mathbb{Q}_{n}^{\text {errw }}\left[\tilde{x}_{e} \geq M\right]=\mathbb{Q}_{n}^{\text {errw }}\left[x_{e} \geq M x_{e_{0}}\right] \leq c_{3}(K) l M^{-\gamma}
$$

and

$$
\mathbb{Q}_{n}^{\text {errw }}\left[\tilde{x}_{e} \leq M^{-1}\right]=\mathbb{Q}_{n}^{\text {errw }}\left[x_{e_{0}} \geq M x_{e}\right] \leq c_{3}(K) l M^{-\gamma} .
$$

This implies tightness of $\mathbb{Q}_{n}^{\text {errw }}\left[\left(\ln \tilde{x}_{e}\right)_{e \in E_{k}} \in \cdot\right], n \geq n_{0}$.

Using a compactness argument, the tightness proven above allows us to construct, by recursion over $k$, a sequence of strictly increasing sequences $m_{k}=\left(m_{k}(i)\right)_{i \in \mathbb{N}}, k \in \mathbb{N}$, with values in $\mathbb{N}$, such that for all $k \in \mathbb{N}, m_{k+1}$ is a subsequence of $m_{k}$ and the sequence $\mathbb{Q}_{m_{k}(i)}^{\text {errw }}\left[\left(\tilde{x}_{e}\right)_{e \in E_{k}} \in \cdot\right]$ converges weakly as $i \rightarrow \infty$. By (5.17), the limiting distribution is supported on $(0, \infty)^{E_{k}}$. Then, the diagonal sequence $n(k):=m_{k}(k), k \in \mathbb{N}$, fulfills the claim of the lemma.

Proof of Theorem 2.2. Let $\mathbb{Q}_{0, a}^{G}$ be the limit of a weakly convergent subsequence $\left(\mathbb{Q}_{n(k)}^{\text {errw }}\left[\left(\tilde{x}_{e}\right)_{e \in E_{k}} \in \cdot\right]\right)_{k \in \mathbb{N}}$ as in Lemma 5.3. By Lemma 5.3, $\mathbb{Q}_{\mathbf{0}, a}^{G}$ is supported on $(0, \infty)^{E}$. 
Let $\pi=\left(\mathbf{0}, v_{1}, \ldots, v_{l}\right)$ be a finite path in $G$. Then, for all $k$ sufficiently large,

$$
\begin{aligned}
P_{\mathbf{0}, a}^{G}\left[\left(X_{s}\right)_{s=0 \ldots l}=\pi\right] & =P_{\mathbf{0}, a}^{G_{n(k)}}\left[\left(X_{s}\right)_{s=0 \ldots l}=\pi\right] \\
& =\int Q_{\mathbf{0}, \tilde{x}}^{G_{n(k)}}\left[\left(X_{s}\right)_{s=0 \ldots l}=\pi\right] \mathbb{Q}_{n(k)}^{\mathrm{errw}}(d x) .
\end{aligned}
$$

Note that for all $k$ large enough and all $x \in(0, \infty)^{E}$, we have $Q_{\mathbf{0} \tilde{x}}^{G_{n(k)}}\left[\left(X_{s}\right)_{s=0 \ldots l}=\pi\right]=$ $Q_{\mathbf{0}, \tilde{x}}^{G}\left[\left(X_{s}\right)_{s=0 \ldots l}=\pi\right]$. Since $x \mapsto Q_{\mathbf{0}, \tilde{x}}^{G}\left[\left(X_{s}\right)_{s=0 \ldots l}=\pi\right]$ is a bounded and continuous cylinder function, taking the limit as $k \rightarrow \infty$ in (5.18) yields

$$
P_{\mathbf{0}, a}^{G}\left[\left(X_{s}\right)_{s=0 \ldots k}=\pi\right]=\int Q_{\mathbf{0}, \tilde{x}}^{G}\left[\left(X_{s}\right)_{s=0 \ldots l}=\pi\right] \mathbb{Q}_{\mathbf{0}, a}^{G}(d x)
$$

The events of the form $\left\{\left(X_{s}\right)_{s=0 \ldots l}=\pi\right\}$ form a closed system with respect to intersection and generate the canonical $\sigma$-algebra on $\Omega_{\mathbf{0}}$. Thus, the claim (2.2) follows from (5.19).

Proof of Theorem 2.3 and 2.4. By Lemmas 5.1 and 5.2, the claimed estimates hold for the measures $\mathbb{Q}_{n(k)}^{\text {errw }}\left[\left(\tilde{x}_{e}\right)_{e \in E_{k}} \in \cdot\right]$, uniformly in $k \in \mathbb{N}$. Lemma 5.3 allows us to take the limit as $k \rightarrow \infty$ to obtain

$$
\mathbb{Q}_{\mathbf{0}, a}^{G}\left[\frac{x_{e}}{x_{v}}<\varepsilon\right] \leq c_{1} \varepsilon^{a_{e} / 2} \quad \text { and } \quad \mathbb{Q}_{\mathbf{0}, a}^{G}\left[\frac{x_{e}}{x_{v}}>1-\varepsilon\right] \leq c_{2} \varepsilon^{\left(a_{v}-a_{e}\right) / 2}
$$

for all $\varepsilon>0$. Taking the limit $\varepsilon \downarrow \tilde{\varepsilon}$, the claims (2.3) follow with $\varepsilon$ replaced by $\tilde{\varepsilon}$. An analogous argument yields (2.5).

Proof of Theorem 2.1. By Theorem 2.2, the edge-reinforced random walk is a mixture of irreducible Markov chains. Since for every irreducible Markov chain the statements (a)(d) are equivalent, the claim follows.

\section{References}

[CD86] D. Coppersmith and P. Diaconis. Random walk with reinforcement. Unpublished manuscript, 1986.

[DF80] P. Diaconis and D. Freedman. de Finetti's theorem for Markov chains. Ann. Probab., 8(1):115-130, 1980.

[Dur04] R. Durrett. Probability: Theory and Examples. Duxbury advanced series, Third edition, 2004.

[KR00] M.S. Keane and S.W.W. Rolles. Edge-reinforced random walk on finite graphs. In Infinite dimensional stochastic analysis (Amsterdam, 1999), pages 217-234. R. Neth. Acad. Arts Sci., Amsterdam, 2000. 
[MR05a] F. Merkl and S.W.W. Rolles. Asymptotic behavior of edge-reinforced random walks. Preprint. Available from http://www.win.tue.nl/〜ssolles, 2005.

[MR05b] F. Merkl and S.W.W. Rolles. Edge-reinforced random walk on a ladder. To appear in The Annals of Probability, 2005.

[MR05c] F. Merkl and S.W.W. Rolles. Linearly edge-reinforced random walks. To appear in Dynamics and Stochastics: Festschrift in the honor of Michael Keane, 2005.

[Rol03] S.W.W. Rolles. How edge-reinforced random walk arises naturally. Probab. Theory Related Fields, 126(2):243-260, 2003.

[Rol05] S.W.W. Rolles. On the recurrence of edge-reinforced random walk on $\mathbb{Z} \times G$. To appear in Probability Theory and Related Fields, 2005.

[Str65] V. Strassen. The existence of probability measures with given marginals. Ann. Math. Statist, 36:423-439, 1965. 